\title{
Proteomic Analysis Reveals the Association between the Rho-GDI Signaling Pathway and Kidney Injury in Spontaneously Hypertensive and Hyperlipidemic Rats
}

\author{
Yasuhiro Taki ${ }^{1}$, Masayuki Oda $^{2}$, Anna Sedukhina ${ }^{2}$, Atsuko Ikemori ${ }^{1,3}$, \\ Naoki Matsumoto ${ }^{4}$, Yugo Shibagaki ${ }^{1}$, and Toshio Kumai ${ }^{2}$
}

(Received for Publication: July 25, 2015)

\begin{abstract}
Chronic kidney disease (CKD) is characterized by progressive, irreversible kidney damage, and the number of patients with CKD has been increasing worldwide. Several studies suggested that hypertension, dyslipidemia, and diabetes are risk factors for CKD. In this study, we generated spontaneously hypertensive and hyperlipidemic rats (SHHRs). In SHHRs, total cholesterol and plasma glucose levels become elevated when fed a highfat, 30\% sucrose diet (HFDS). However, the molecular mechanism underlying the effect of hypertensive, dyslipidemic, and hyperglycemic conditions on the kidney remains unknown. To elucidate the mechanism, we performed proteomic analysis of the kidney in SHHRs. Four-month-old male Sprague-Dawley (SD) rats and SHHRs were fed a normal diet (ND) or HFDS ad libitum for 6 months. Proteins in the renal cytoplasm were separated by LC/MS, and proteomic analysis was performed. Differentially expressed proteins were linked using Ingenuity Pathway Analysis (IPA). We identified Rho-GDP dissociation inhibitor (Rho-GDI) signaling as a candidate pathway involved in kidney injury in SHHRs with HFDS feeding. In the kidney, the levels of RhoGDI protein and Rho GTPase-activating protein (Rho-GAP) decreased, and that of p21-activated kinase (PAK) significantly increased. In addition, the renal expression of Arhgdia (encoding Rho-GDI), Dlcl (encoding RhoGAP), and Pakl (encoding PAK) genes were, at least in part, parallel to that of proteins in SHHRs that had been fed the HFDS. Furthermore, neutrophil gelatinase-associated lipocalin ( $\mathrm{Ngal}$ ), a biomarker of kidney injury, was highly expressed in the kidney of SHHRs that had been fed the HFDS. These data suggest the possibility that the Rho-GDI signaling pathway is activated in the kidney of SHHRs that are fed the HFDS, leading to kidney injury. Taken together, our findings provide a molecular basis for the effects of hypertensive, dyslipidemic, and hyperglycemic conditions on the kidney.
\end{abstract}

Key words

kidney, proteomic analysis, SHHR, Rho-GDI signaling

\section{Introduction}

Recently, the number of patients with chronic kidney disease (CKD) has increased, reaching 13.3 million in Japan in $2005^{1)}$. It is well known that CKD directly increases mortality and decreases the ability to perform activities of daily living. Hypertension, dyslipidemia, and diabetes are all risk factors for CKD pathogenesis ${ }^{2-4}$. Hypertension leads to sclerosis of the afferent arterioles and causes a loss of autore-

\footnotetext{
1 Division of Nephrology and Hypertension, Department of Internal Medicine, St. Marianna University School of Medicine, Kawasaki, Japan.

2 Department of Pharmacogenomics, St. Marianna University Graduate School of Medicine, Kawasaki, Japan.

3 Department of Anatomy, St. Marianna University School of Medicine, Kanagawa, Japan

4 Department of Pharmacology, St. Marianna University School of Medicine, Kawasaki, Japan.
} 
gulation in the glomerulus ${ }^{5)}$. Hypertension also causes arteriolar lumen narrowing, resulting in decreased blood flow in the glomerulus and tubulointerstitium ${ }^{5}$. In addition, the deposition of excessive low-density lipoprotein cholesterol can activate glomerular macrophages ${ }^{6}$. These macrophages produce reactive oxygen species and damage vascular endothelial cells and mesangial cells via inflammatory and fibrogenic factors. Furthermore, the increased concentration of intracellular glucose activates a polyol pathway of glucose metabolism, causing kidney injury through the accumulation of advanced glycation end-products and the activation of oxidative stress ${ }^{7}$.

We previously established a spontaneously hypertensive hyperlipidemic rat (SHHR) line by crossing Sprague-Dawley (SD)-derived hyperlipidemic rats with Wistar-Kyoto-derived spontaneously hypertensive rats $(\mathrm{SHRs})^{8}$. SHHRs have systolic blood pressure higher than $150 \mathrm{mmHg}$ and a total cholesterol level greater than $150 \mathrm{mg} / \mathrm{dl}$ when receiving a normal diet. Furthermore, SHHRs experience elevated total cholesterol and plasma glucose levels when fed a high-fat, $30 \%$ sucrose diet (HFDS) $)^{9}$. Therefore, this model is useful for studying physiology, pathogenesis, and pharmacology under hypertensive, hyperlipidemic, and hyperglycemic conditions. Using this rat model, we previously found that visceral fat accumulates in the body and that plasma levels of oxidative stress markers increase in SHHRs fed with HFDS $^{9,10)}$. In addition, Oda et al. showed that the protein expression of glutathione peroxidase, which functions as an antioxidant, decreases in the kidney of SHHRs that are fed the HFDS ${ }^{11}$. These data suggest that oxidative stress accumulates in the kidneys of SHHRs that are fed the HFDS, which could cause kidney injury. However, the molecular mechanism underlying the effects of the hypertensive, hyperlipidemic, and hyperglycemic conditions on the kidney remains unknown. In the present study, we performed proteomic analysis to investigate which molecules in the kidney are involved in hypertension, hyperlipidemia, and hyperglycemia.

\section{Materials and Methods}

\section{Animals}

We used 10-month-old male SD rats and SHHRs for experiments. The rats were housed in a semi-barrier system with controlled room temperature (23 $\pm 1{ }^{\circ} \mathrm{C}$ ), humidity $(55 \pm 5 \%$ ), and lighting (lights on from 6:00 to 18:00). Until the age of 3 months, all rats were fed the normal diet (ND) ad libitum. The
SD rats and SHHRs were then divided into 2 groups each. One group was fed the ND for 7 months, and the other group was orally administered $100 \mathrm{mg} / \mathrm{L}$ of $\mathrm{N}^{\mathrm{G}}$-nitro-L-arginine methyl ester in drinking water for 1 month and fed the HFDS for 6 months. The ND was regular rat chow (CE2; CLEA Japan Inc., Tokyo, Japan) comprised of $8.9 \%$ water, $25.4 \%$ protein, $4.4 \%$ fat, $4.1 \%$ fiber, $6.9 \%$ carbohydrate, and $50.3 \%$ nitrogen-free extracts, containing $342.2 \mathrm{kcal} / 100 \mathrm{~g}$. The high-fat diet consisted of $8.2 \%$ water, $23.4 \%$ protein, $11.0 \%$ fat, $3.8 \%$ fiber, $6.3 \%$ carbohydrate, and 46.3\% nitrogen-free extracts, containing 378.0 $\mathrm{kcal} / 100 \mathrm{~g}$ (CLEA, Japan). After 6-month feeding on the respective diets, the rats were euthanized under pentobarbital anesthesia, and the weight of visceral adipose and epididymal adipose tissue was determined. Serum was obtained from blood samples by centrifugation and used for the measurement of the derivatives of reactive oxygen metabolite (d-ROM) concentrations using the Free Radical Analytical System (Diacron International, Grosseto, Italy). All experiments were conducted according to the Guiding Principles for the Care and Use of Laboratory Animals of St. Marianna University School of Medicine.

\section{Extraction of subcellular proteomes}

Cytosolic and membrane/organelle protein fractions of tissues were isolated from rat kidneys using the ProteoExtract Subcellular Proteome Extraction Kit (Millipore, Billerica, MA, USA) following the manufacturer's procedure. In brief, $50 \mathrm{mg}$ of fragmented kidney was homogenized in $100 \mu \mathrm{L}$ of Extraction Buffer I with Protease Inhibitor Cocktail (Millipore), and incubated for $10 \mathrm{~min}$ at $4{ }^{\circ} \mathrm{C}$ with shaking. The supernatant was separated by centrifugation for $10 \mathrm{~min}$ at $1000 \times \mathrm{g}$ at $4^{\circ} \mathrm{C}$ (as the cytosolic protein fraction). The pellet was resuspended in 300 $\mu \mathrm{L}$ of Extraction Buffer II with Protease Inhibitor Cocktail (Millipore), and incubated for $30 \mathrm{~min}$ at $4{ }^{\circ} \mathrm{C}$ with shaking. The supernatant was separated by centrifugation for $10 \mathrm{~min}$ at $6000 \times \mathrm{g}$ at $4^{\circ} \mathrm{C}$ (as the membrane/organelle protein fraction).

\section{Nano-HPLC/ESI-ion trap-MS/MS analysis}

The supernatants $(5 \mu \mathrm{L})$ obtained from the kidney samples were diluted and analyzed using a nanoHPLC system (1200 Series; Agilent Technologies Inc., Palo Alto, CA, USA), connected to an HCT Ultra ion trap mass spectrometer (Bruker Daltonics, Billerica, MA, USA). A 5CD-C18 trap column (Imtakt, Kyoto, Japan) was used to concentrate and de- 
salt the injected samples. Chromatographic separation of peptides was carried out using a 5CD-C18 analytical column. Sample elution through the analytical column was obtained at a flow rate of 300 $\mathrm{nL} / \mathrm{min}$ by starting the following gradient: $5-40 \%$ solvent B for $100 \mathrm{~min}$; step with $80 \%$ solvent B for 5 min; and step with $5 \%$ solvent B for $15 \mathrm{~min}$. Solvent was $0.1 \%$ formic acid in water, and solvent $\mathrm{B}$ was $0.1 \%$ formic acid in acetonitrile. The HCT Ultra was operated in positive-ion mode with a maximum accumulation time of $200 \mathrm{~ms}$ in an $\mathrm{m} / \mathrm{z}$ range of 350 1300. The auto-MS/MS analysis was carried out with 3 precursor ions, an absolute threshold of 1000, and an active ion precursor exclusion for $0.5 \mathrm{~min}$ after two MS/MS spectra were obtained. The peptides were fragmented in the CID mode. Fragmentation parameters were as follows: width $4.0 \mathrm{~m} / \mathrm{z}$; and time of fragmentation $200 \mathrm{~ms}$. The mass spectrometry data obtained were analyzed using MASCOT software (Matrix Science, London, UK).

\section{Ingenuity Pathway Analysis}

Proteomic analysis data were analyzed with Ingenuity Pathway Analysis (IPA) software following the manufacturer's procedure (http:// www.ingenuity.com/). The significance of the common networks and canonical pathways were tested based on the $p$-value. The associative networks were shown based on the score.

\section{Quantitative RT-PCR analysis}

Total RNA was extracted from the specimens using the RNeasy Mini Kit (Qiagen, Frederick, MD, USA), following the manufacturer's instructions. A RETROscript Kit (Ambion, Austin, TX, USA) was used to synthesize cDNA from total RNA. cDNA was amplified with the LightCycler SYBR Green Master mix using a LightCycler (Roche Diagnostics, Mannheim, Germany). Fluorescence data were analyzed with LightCycler software (Roche Diagnostics). Expressions of target genes were normalized to Gapdh mRNA levels measured concurrently. The primer list is given in Table 1.

\section{Statistical analysis}

For IPA, the $p$-value was calculated using Fisher's exact test. The significance of differences among groups was determined by the analysis of variance (ANOVA) Tukey-Kramer post hoc test. A $p$-value of 0.05 was considered to represent a statistically significant difference.
Table 1. Primer Pairs for RT-PCR Analysis.

\begin{tabular}{cll} 
Gene & \multicolumn{2}{c}{ Primer sequence } \\
\hline \multirow{2}{*}{ Arhgdia } & Forward & CAATGTCCCCAACGTCATTGTGACC \\
& Reverse & TTATCCGGTACTCCACACCTTCCTT \\
DIc1 & $\begin{array}{l}\text { Forward } \\
\text { Reverse }\end{array}$ & AGTTTGGGCAAACCAGACCA \\
& GGCTCAGCTCCTGTTCAGT \\
Pak1 & Forward & ATGCTTTGACCCGGAATACT \\
& Reverse & TGAAGCACCTTGTCCAATCT \\
Ngal & Forward & ACTCAACTCAGACTTGATCCCTGC \\
& Reverse & AAGCGGCTTTGTCTTTCTTTCTGGA \\
Gapdh & Forward & CTGAGTATGTCGTGGAGTCTA \\
& Reverse & CTGCTTCACCACCTTCTTGAT
\end{tabular}

\section{Results}

\section{Biological studies of SD rats and SHHRs.}

Body weight and kidney weight did not change in either SD rats or SHHRs (Fig. 1A, 1B). Visceral adipose tissue did not increase in SD rats that were fed the HFDS (SD-HFDS group), while it was significantly increased in SHHRs fed the same diet (SHHR-HFDS group) (Fig. 1C). The amount of epididymal adipose tissue did not change in either SD rats or SHHRs on the HFDS (Fig. 1D). The oxidative stress marker d-ROM significantly increased in serum in the HFDS groups, although there was no difference between SD rats and SHHRs (Fig. 1E).

\section{Proteomic analysis identified Rho-GDI signaling in the kidney of SHHRs.}

When proteomic analysis and IPA were performed to investigate the effects of hypertension, dyslipidemia, and hyperglycemia on the SHHR kidney, the results showed that the level of Rho-GDI protein decreased, and that of p21-activated kinase (PAK) increased in the kidney of SD-HFDS compared with SD-ND (Table 2). The similar changes in the expressions of Rho-GDI and PAK proteins were also observed in SHHR-HFDS in comparison with SDHFDS. In addition, the protein expression of Rho GTPase-activating protein (Rho-GAP) was downregulated in the kidney of SHHRs when fed HFDS. In the category of injury of kidney, Rho-GDI protein expression decreased in the SHHR-HFDS group (Table 3). Since the ratio, referring to the percentage of hit proteins in the Rho-GDI signaling pathway was the highest when comparing SHHR-ND with SHHRHFDS, we performed IPA focusing on the comparison between these groups. Rho-GDI signaling, cardiomyocyte differentiation via bone morphogenetic protein (BMP) receptors, and vascular endothelial 


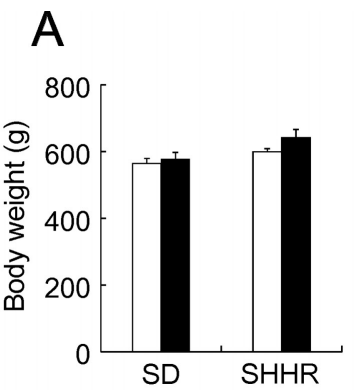

\section{B}
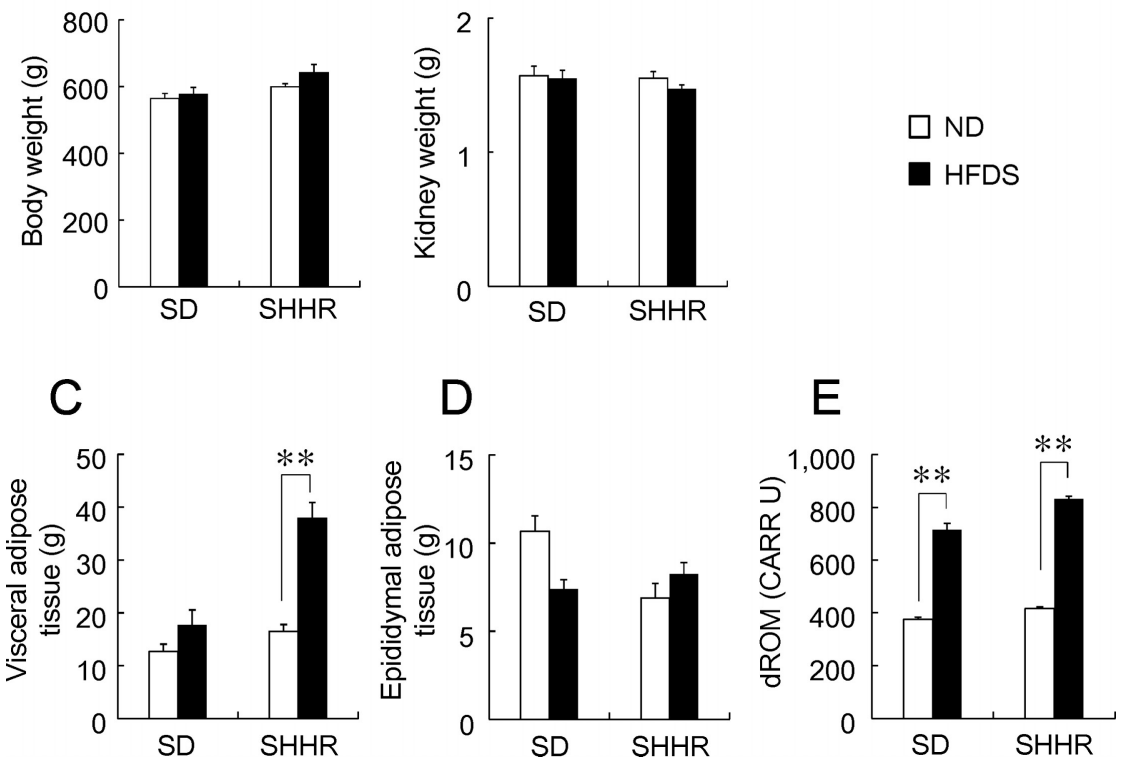

Fig. 1. Effects of HFDS on the body weights, the weights of kidney, visceral adipose tissue, and epididymal adipose tissue, and the serum concentrations of d-ROMs SD rats and SHHRs.

SD rats and SHHRs were fed either the ND or HFDS for 6 months. The body weight (A), and the weights of the kidney (B), visceral adipose tissue $(\mathbf{C})$, and epididymal adipose tissue (D) was determined. (E) The concentration of d-ROMs in serum was measured using a commercial kit. Each value represents mean $\pm \mathrm{SE}(n=7)$. $* * P<$ 0.01 , Tukey-Kramer post hoc test.

Table 2. Highly scored proteins in the Rho-GDI signaling pathway.

\begin{tabular}{ccccc} 
Canonical pathway & \multicolumn{2}{c}{ Subtype } & Molecule & $p$-value \\
\cline { 2 - 3 } & $(1)$ vs $(2)$ & Rho-GDI $(\downarrow)$, PAK $(\uparrow)$ & 1.23 E-03 \\
& SD ND vs SD HFDS & - & - \\
Rho-GDI signaling & SD ND vs SHHR ND & SD HFDS vs SHHR HFDS & Rho-GDI $(\downarrow), \operatorname{PAK}(\uparrow)$ & $1.23 E-03$ \\
& SHHR ND vs SHHR HFDS & Rho-GDI $(\downarrow), \operatorname{Rho-GAP}(\downarrow), \operatorname{PAK}(\uparrow)$ & $1.34 \mathrm{E}-03$
\end{tabular}

Differentially expressed proteins associated with Rho-GDI signaling are listed. Upward arrows show upregulated proteins and downward arrows show downregulated proteins in the renal cytoplasm of (2) compared with that of (1). The $p$-value was calculated using Fisher's exact test.

growth factor (VEGF) signaling were the top 3 most significant signaling pathways that differed between the SHHR-ND and SHHR-HFDS groups (Fig. 2). These results indicate that Rho-GDI signaling would be important in the kidney under hypertensive, dyslipidemic, and hyperglycemic conditions. We next performed pathway analysis for Rho-GDI signaling. The protein expression of Rho-GDI and Rho-GAP de- creased, and that of PAK increased in the kidney in the SHHR-HFDS group (Fig. 3). These data suggest that GTP-bound forms of Rho-GTPases would increase and activate downstream signaling in the kidneys of SHHRs fed the HFDS. 
Table 3. Highly Scored Proteins in the Category of Injury of Kidney.

\begin{tabular}{|c|c|c|c|}
\hline \multirow{2}{*}{ Category } & Subtype & \multirow{2}{*}{ Molecules } & \multirow{2}{*}{$p$-value } \\
\hline & (1) vs (2) & & \\
\hline \multirow{4}{*}{ Injury of kidney } & SD ND vs SD HFDS & Rho-GDI $(\downarrow)$, catalase $(\downarrow)$ & $7.22 \mathrm{E}-03$ \\
\hline & SD ND vs SHHR ND & Catalase $(\downarrow)$ & $3.41 \mathrm{E}-03$ \\
\hline & SD HFDS vs SHHR HFDS & Rho-GDI $(\downarrow)$, catalase $(\downarrow)$ & $7.22 \mathrm{E}-03$ \\
\hline & SHHR ND vs SHHR HFDS & Rho-GDI $(\downarrow)$, catalase $(\downarrow)$ & $7.22 \mathrm{E}-03$ \\
\hline
\end{tabular}

Differentially expressed proteins included in the category of injury of kidney are listed. Downward arrows show downregulated proteins in the renal cytoplasm of (2) compared with that of (1). The $p$-value was calculated using Fisher's exact test.

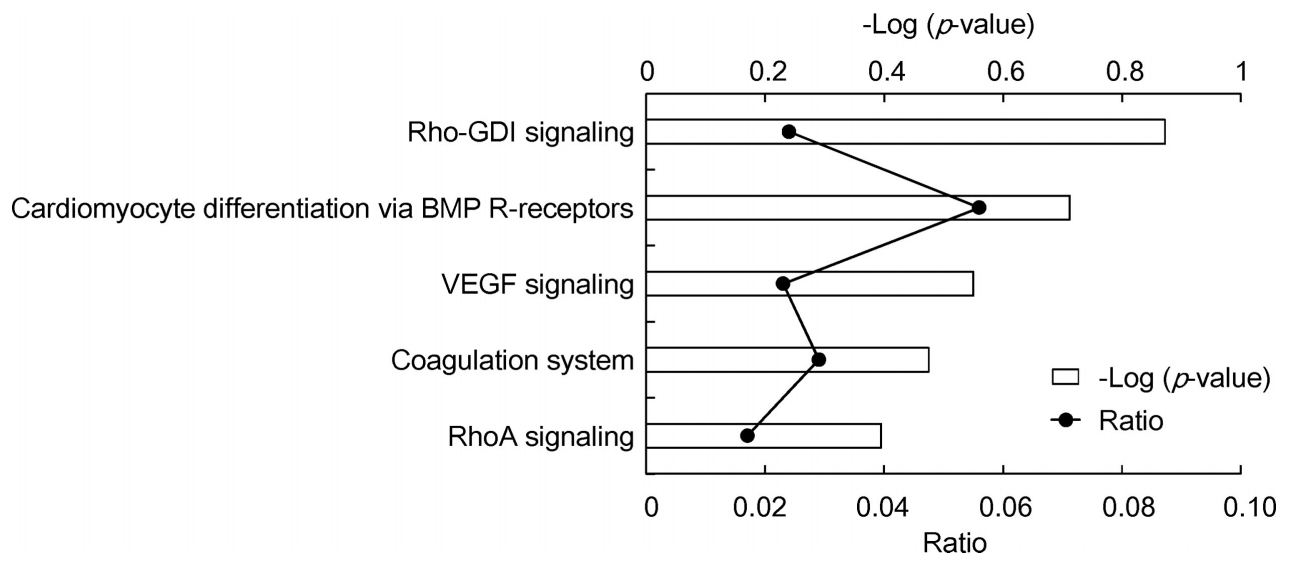

Fig. 2. Highly scored canonical pathways in the kidney of SHHRs fed the ND vs HFDS by IPA.

IPA was performed using LC/MS data in the kidney cytoplasm of SHHRs fed either the ND or HFDS. The $p$-value was calculated using Fisher's exact test. The ratio shows the number of differentially expressed proteins involved in the pathway divided by the total number of proteins making up that pathway.

Gene expression of Arhgdia, Dlc1, and Pak1 in the kidney of SD rats and SHHRs.

Proteomic analysis identified Rho-GDI, RhoGAP, and PAK as candidate molecules affecting the kidney under hypertensive, dyslipidemic, and hyperglycemic conditions. To investigate whether the expression of these proteins is regulated at the transcriptional level, we measured the gene expression of Arhgdia (encoding Rho-GDI), Dlcl (encoding RhoGAP), and Pakl (encoding PAK) using real-time PCR analysis. The expression of Arhgdia mRNA significantly decreased in the kidney in the SHHRHFDS group compared with the other groups (Fig. 4A). The gene expression of $D l c l$ exhibited a significant decrease in both the SD-HFDS and SHHRHFDS groups (Fig. 4B). These changes in mRNA expression were partially similar to those of proteins (Table 2), thereby indicating that the expression of
Rho-GDI and Rho-GAP proteins would be regulated at both transcriptional and translational levels. On the other hand, the gene expression of Pakl was parallel to its protein expression in the kidney (Fig. 4C, Table 2), suggesting that the expression of PAK would be driven at the transcriptional level in SHHRs fed the HFDS.

\section{High expression levels of $\mathrm{Ngal}$ in the kidney of SHHRs fed the HFDS.}

Because IPA pointed to the possibility that RhoGDI signaling is activated in the kidney of SHHRs fed the HFDS, we measured the gene expression of $\mathrm{Ngal}$ in the kidney. $\mathrm{Ngal}$ gene expression significantly increased in the kidneys in the SHHR-HFDS group (Fig. 5). 


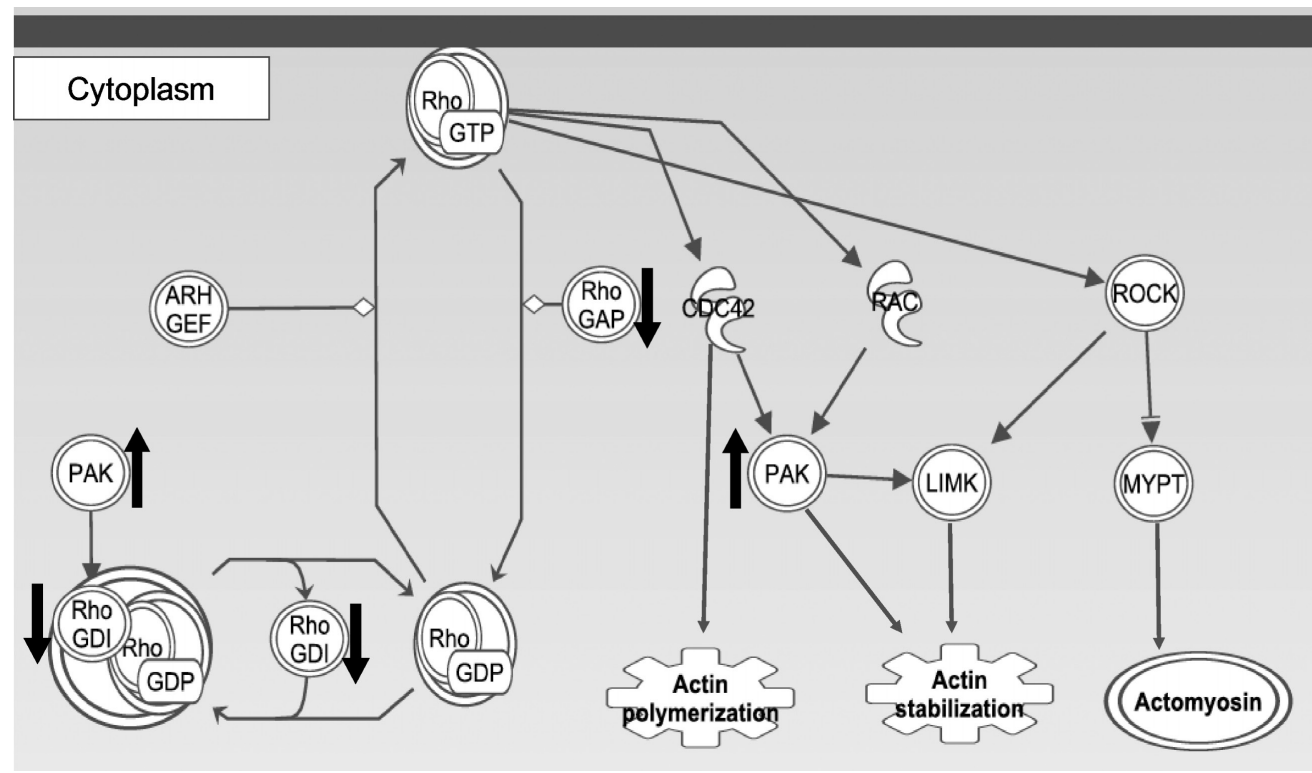

Fig. 3. IPA results indicate the enhancement of Rho-GTPase activity in the kidney of SHHRs fed the HFDS. The Rho-GDI signaling pathway shown by IPA is illustrated by differentially expressed proteins. Upward black arrows show upregulated proteins and downward black arrows show downregulated proteins in the kidney cytoplasm of the SHHR-HFDS compared with the SHHR-ND group.

A

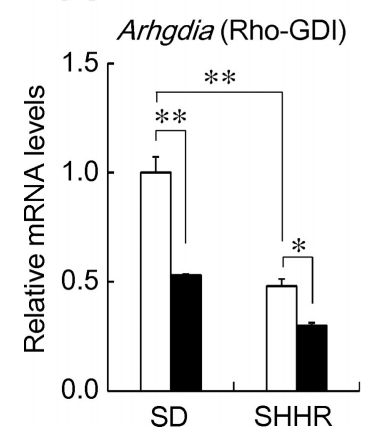

B

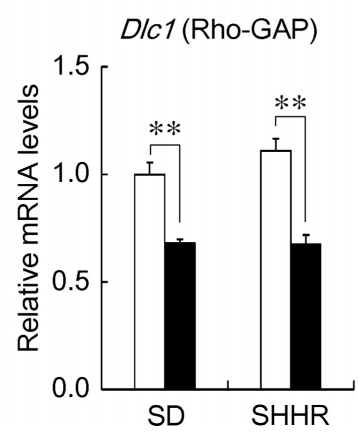

C

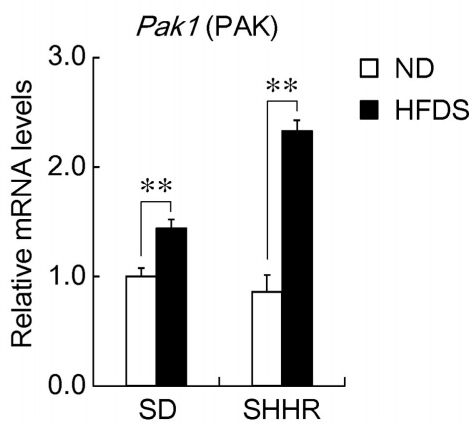

Fig. 4. Effects of HFDS on the gene expression of Arhgdia, Dlc1, and Pak1 in the kidney of SD rats and SHHRs.

The mRNA expression of (A) Arhgdia, (B) Dlcl, and (C) Pakl was determined using quantitative PCR analysis. Data were normalized to the level of Gapdh mRNA expression. Each value represents mean $\pm \operatorname{SE}(n=7)$. $* P<0.05$, $* * P<0.01$, Tukey-Kramer post hoc test.

\section{Discussion}

In this study, we performed proteomic analysis using the kidney of SHHRs to investigate the effect of hypertensive, dyslipidemic, and hyperglycemic conditions on the kidney. We first measured biological parameters, such as body weight, weights of kidney and visceral adipose tissue, and dROM in serum.
These parameters tended to be similar to those in a previous study ${ }^{10)}$. Although the values of dROM were a little different from previous reports ${ }^{10)}$, Oda et al. administered HFDS to rats for 4 months, while we administered for 6 months. Thus, the different values of dROM may be related to the difference in duration of HFDS. The results of the proteomic analysis in, our present study suggest that kidney injury could be 


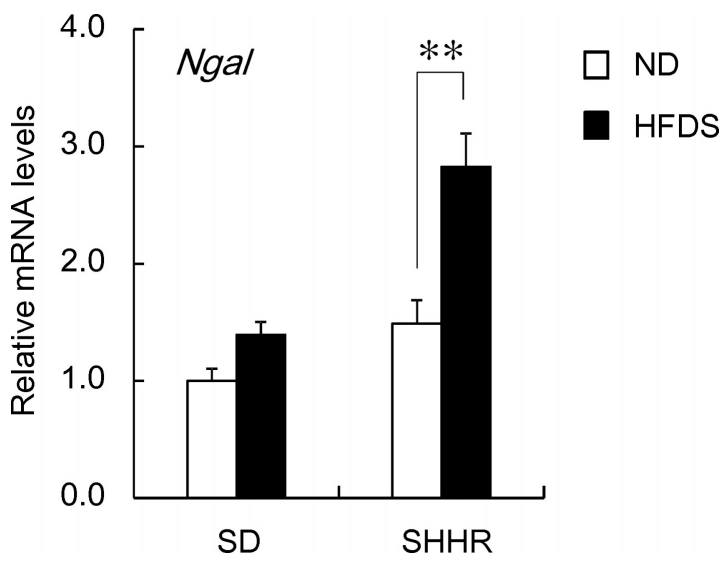

Fig. 5. Effects of HFDS on Ngal gene expression in the kidney of SD rats and SHHRs.

The mRNA expression of Ngal was determined using quantitative PCR analysis. Data were normalized to the level of Gapdh mRNA expression. Each value represents mean $\pm \operatorname{SE}(n=7)$. $* * P<0.01$, Tukey-Kramer post hoc test.

induced through Rho-GDI signaling in SHHRs fed the HFDS. Rho proteins are guanine nucleotide-binding proteins that belong to the large group of GTPases and widely involved in many biological functions ${ }^{11}$. G proteins, such as Rho-GTPases, are molecular switches that cycle between an inactive GDP-bound and an active GTP-bound conformation. To turn on the switch, GTP loading to Rho-GTPases is facilitated by Rho-specific guanine nucleotide exchange factors (Rho-GEFs). Conversely, GTP hydrolysis is catalyzed by Rho-GAP to turn off the switch. Rho-GDIs dissociate inactive Rho-GTPases from the cell membrane to prevent them from misfolding and degrading, resulting in the inhibition of Rho-GTPase activities $^{12}$. On the other hand, PAK is the effector of RAC1 and CDC42 GTPases, which are Rho-GTPase family members ${ }^{13)}$ Thus, PAK stimulates the downstream signaling of RAC1 and CDC42 GTPases. In SHHRs, the protein expression of Rho-GDI and RhoGAP was decreased, and that of PAK was increased, in the kidney in the HFDS groups. These findings indicate that levels of the GTP-bound forms of RhoGTPases would be increased, activating the downstream signaling in the kidney of SHHRs fed the HFDS. Previous studies revealed that the activation of Rho-GTPase leads to kidney injury ${ }^{14-16)}$. The loss of Rho-GDI causes progressive kidney defects that are attributable to increased RAC1 activity, ultimately leading to death in mice ${ }^{15)}$. The gene expression of
Ngal, a biomarker of kidney injury ${ }^{17)}$, was significantly increased in the kidney in the SHHR-HFDS group. The observation indicates that the kidney injury would be mediated through Rho-GDI signaling in SHHRs fed the HFDS. Interestingly, a recent study has demonstrated that Arhgdia mutations (R120X and G173V) cancel interaction with Rho-GTPases and increase GTP-bound forms of RAC1 and CDC42, but not RhoA, in patients with steroid-resistant nephrotic syndrome ${ }^{18)}$. Furthermore, entire exome sequencing in two sisters with congenital nephrotic syndrome revealed that mutant Rho-GDI produced by Arhgdia with an in-frame deletion (D185del) is unable to bind to Rho-GTPases ${ }^{19}$. These reports support our observations that the expression of Rho-GDI decreases in the kidneys of SHHRs fed the HFDS, with concomitant kidney damage. However, we did not show the data about the direct relationship between NGAL and Rho-GDI signaling. A certification study will be needed.

It was also shown that the activation of RhoGDI signaling is linked with hypertension, dyslipidemia, and diabetes ${ }^{20-22)}$. For example, RhoA kinase (ROCK) activity is enhanced in the aorta obtained from spontaneously hypertensive rats $^{20)}$. The increased ROCK activity is significantly suppressed by the antidiabetic agent pioglitazone, accompanied by a reduction in blood pressure ${ }^{20)}$. Furthermore, another study suggested that simvastatin, a lipid-lowering agent, inhibits high glucose-induced RhoGTPase/p21 signaling in glomerular mesangial cells, implying the potential for its therapeutic use in diabetic nephropathy ${ }^{21)}$. However, no report has identified the role of Rho-GDI signaling in the kidney under hypertensive, dyslipidemic, and hyperglycemic conditions through comprehensive analysis. The present proteomic analysis emphasizes the importance of Rho-GDI signaling in the kidney of SHHRs.

In conclusion, we found that kidney injury is mediated through Rho-GDI signaling in SHHRs fed the HFDS. These findings provide insight into the molecular basis underlying the effects of hypertensive, dyslipidemic, and hyperglycemic conditions on the kidney. CKD may be prevented by ameliorating these conditions and by novel therapies targeting molecules in the Rho-GDI signaling pathway.

\section{Acknowledgement}

We would like to express our sincere appreciation to Dr. Shinichi Iwai for his valuable advice and technical support. We would like to thank Dr. Yuko 
Takeba and Dr. Ko Sato for their useful advice. We are grateful to Dr. Hiroyuki Nishikawa and Dr. Shigeko Oonuma for their technical support.

\section{References}

1) Japanese Society of Nephrology. Special issue: Clinical practice guidebook for diagnosis and treatment of chronic kidney disease 2012. Nihon Jinzo Gakkai Shi 2012; 54: 1031-1191.

2) Klag MJ, Whelton PK, Randall BL, Neaton JD, Brancati FL, Ford CE, Shulman NB, Stamler J. Blood pressure and end-stage renal disease in men. N Engl J Med 1996; 334: 13-18.

3) Schaeffner ES, Kurth T, Curhan GC, Glynn RJ, Rexrode KM, Baigent C, Buring JE, Gaziano JM. Cholesterol and the risk of renal dysfunction in apparently healthy men. J Am Soc Nephrol 2003; 14: 2084-2091.

4) Adler AI, Stevens RJ, Manley SE, Bilous RW, Cull CA, Holman RR; UKPDS GROUP. Development and progression of nephropathy in type 2 diabetes: The United Kingdom Prospective Diabetes Study (UKPDS 64). Kidney Int 2003; 63: 225-232.

5) Kramer H, Luke H, Bidani A, Cao G, Cooper R, McGee D. Obesity and Prevalent and Incident CKD: The Hypertension Detection and FollowUp Program. Am J kidney Dis 2005; 46: 587594.

6) Esposito K, Pontillo A, Di Palo C, Giuglinano G, Masella M, Marfella R, Giugliano D. Effect of weight loss and lifestyle changes on vascular inflammatory markers in obese women: a randomized trial. JAMA 2003; 289: 1799-1804.

7) Cachofeiro V, Goicochea M, de Vinuesa SG, Oubina P, Lahera V, Luno J. Oxidative stress and inflammation, a link between chronic kidney disease and cardiovascular disease. Kidney Int Suppl 2008; 111: S4-S9.

8) Kumai T, Oonuma S, Kitaoka Y, Tadokoro M, Kobayashi S. Biochemical and morphological characterization of spontaneously hypertensive hyperlipidemic rats. Clin Exp Pharmacol Physiol 2003; 30: 537-544.

9) Saiki R, Okazaki M, Iwai S, Kumai T, Kobayashi S, Oguchi K. Effects of Pioglitazone on Increases in Visceral Fat Accumulation and Oxidative Stress in Spontaneously Hypertensive Hyperlipidemic Rats Fed a High-Fat Diet and Sucrose Solution. J Pharmacol Sci 2007; 105: 157-167.
10) Oda G, Shirai S, Shibagaki Y, Ohnuma S, Koike J, Matsumoto N, Kimura K, Kumai T. The Change of Oxidative Stress Scavenger System of the Kidney in High Fat Diet and Sucrose-treated Spontaneously Hypertensive Hyperlipidemic Rats. The St. Marianna Medical Journal 2014; 41: 289-298.

11) Iden S, Collard JG. Crosstalk between small GTPases and polarity proteins in cell polarization. Nat Rev Mol Cell Biol 2008; 9: 846-859.

12) Garcia-Mata R, Boulter E, Burridge K. The 'invisible hand': regulation of RHO GTPases by RHOGDIs. Nat Rev Mol Cell Biol 2011; 12: 493-504.

13) Zhao ZS, Manser E. PAK and other Rho-associated kinases-effectors with surprisingly diverse mechanisms of regulation. Biochem J 2005; 386: 201-214.

14) Babelova A, Jansen F, Sander K, Löhn M, Schäfer L, Fork C, Ruetten H, Plettenburg O, Stark H, Daniel C, Amann K, Pavenstädt H, Jung O, Brandes RP. Activation of Rac-1 and RhoA contributes to podocyte injury in chronic kidney disease. Plos One 2013; 8: e80328.

15) Shibata S, Nagase M, Yoshida S, Kawarazaki W, Kurihara H, Tanaka H, Miyoshi J, Takai Y, Fujita T. Modification of mineralocorticoid receptor function by Rac1 GTPase: implication in proteinuric kidney disease. Nat Med 2008; 14: 1370-1376.

16) Yu H, Suleiman H, Kim AH, Miner JH, Dani A, Shaw AS, Akilesh S. Rac1 Activation in Podocytes Induces Rapid Foot Process Effacement and Proteinuria. Mol Cell Biol 2013; 33: 47554764.

17) Mishra J, Dent C, Tarabishi R, Mitsnefes MM, Ma Q, Kelly C, Ruff SM, Zahedi K, Shao M, Bean J, Mori K, Barasch J, Devarajan P. Neutrophil gelatinase-associated lipocalin (NGAL) as a biomarker for acute renal injury after cardiac surgery. Lancet 2005; 365: 1231-1238.

18) Gee HY, Saisawat P, Ashraf S, Hurd TW, VegaWarner V, Fang H, Beck BB, Gribouval O, Zhou W, Diaz KA, Natarajan S, Wiggins RC, Lovric S, Chernin G, Schoeb DS, Ovunc B, Frishberg Y, Soliman NA, Fathy HM, Goebel H, Hoefele J, Weber LT, Innis JW, Faul C, Han Z, Washburn J, Antignac C, Levy S, Otto EA, Hildebrandt F. ARHGDIA mutations cause nephrotic syndrome via defective RHO GTPase signaling. J Clin Invest 2013; 123: 3243-3253. 
19) Gupta IR, Baldwin C, Auguste $\mathrm{D}, \mathrm{Ha} \mathrm{KC}, \mathrm{El}$ Andalousi J, Fahiminiya S, Bitzan M, Bernard C, Akbari MR, Narod SA, Rosenblatt DS, Majewski J, Takano T. ARHGDIA: a novel gene implicated in nephrotic syndrome. J Med Genet 2013; 50: 330-338.

20) Wakino S, Hayashi K, Kanda T, Tatematsu S, Homma K, Yoshioka K, Takamatsu I, Saruta T. Peroxisome proliferator-activated receptor gamma ligands inhibit Rho/Rho kinase pathway by inducing protein tyrosine phosphatase SHP-2. Circ Res 2004; 95: e45-e55.

21) Danesh FR, Sadeghi MM, Amro N, Philips C,
Zeng L, Lin S, Sahai A, Kanwar YS. 3-Hydroxy-3-methylglutaryl CoA reductase inhibitors prevent high glucose-induced proliferation of mesangial cells via modulation of Rho GTPase/ p21 signaling pathway: Implications for diabetic nephropathy. Proc Natl Acad Sci U S A 2002; 99: 8301-8305.

22) Tabur S, Oztuzcu S, Oguz E, Korkmaz H, Eroglu S, Ozkaya M, Demiryürek AT. Association of Rho/Rho-kinase gene polymorphisms and expressions with obesity-related metabolic syndrome. Eur Rev Med Pharmacol Sci 2015; 19 : 1680-1688. 